Research Article

\title{
Experimental Evaluation of the Accuracy of Biped Model to Predict the Kinematics during Walking
}

\author{
Yao Zhang $\mathbb{D}^{1,2}$ and Zhigang Song $\mathbb{D}^{1}$ \\ ${ }^{1}$ Kunming University of Science and Technology, Kunming 650500, China \\ ${ }^{2}$ College of Applied Sciences, Qujing Normal University, Qujing 655000, China \\ Correspondence should be addressed to Zhigang Song; zhigang-s@163.com
}

Received 21 November 2020; Revised 26 March 2021; Accepted 1 April 2021; Published 19 April 2021

Academic Editor: Roberto Nascimbene

Copyright (c) 2021 Yao Zhang and Zhigang Song. This is an open access article distributed under the Creative Commons Attribution License, which permits unrestricted use, distribution, and reproduction in any medium, provided the original work is properly cited.

\begin{abstract}
Biped models (BMs) have extensive applications in structural vibration, robotics, and sports medicine, but the differences between the walking kinematics simulated by the BM model and those measured through an experiment have not been comprehensively recognized. This paper used wireless inertial measurement units (IMUs) to monitor the acceleration of center of masses (COMs) of 6 volunteers walking under the conditions of natural and fixed-knee gait. The observations were compared with the simulations from two typical BMs and also with the measurements from former research studies. The results show that when the step frequency is lower than $2.0 \mathrm{~Hz}$, the peaks of acceleration simulated by BM are close to those observed in experiments and the errors are acceptable, while the step frequency is higher than $2.0 \mathrm{~Hz}$, the discrepancy between the numerical and the experimental results is increasingly larger with higher step frequencies, and the errors can reach $60 \%$ at most. The comparison between numerical and experimental results indicates that the knee-fixing assumption of BMs may lead to overestimating the peaks of accelerations, but this assumption fails to explain the different increasing rates with respect to step frequency. Further research studies about the accuracy of BMs and relative modification methods are expected for civil engineering applications.
\end{abstract}

\section{Introduction}

Biomechanic models that describe human walking patterns have been widely adopted in multidisciplines including civil engineering, robotics, and sports medicine [1-5]. In structural engineering, these models are typically adopted to estimate the dynamic loads induced by human to civil structures $[6,7]$. Based on the anticipated loads, serviceability of structures could be evaluated during design.

The biped model (BM) [8,9], a typical biomechanic model originated from the inverted pendulum model [10], is capable of illustrating a sequence of walking and running steps. It describes the kinematic features of center of mass (COM), the alternation between single and double leg support phase, and the $\mathrm{M}$-shaped force profile of a single footfall with a small amount of parameters. The BM simulations require less computation efforts than other complex models and have been widely applied by several gait analyses of the pedestrians with varying ages and carrying different levels of loads $[11,12]$.

The primitive biped model (PBM) proposed by Geyer et al. [8] mainly has faced two difficulties in describing the kinematic and kinetic features of human walking: the first is maintaining the steady-state walking, while the energy dissipation of damping is introduced; the second is that the magnitudes of the COM oscillation and the ground reaction force (GRF) predicted by PBM are remarkably higher than that under practical observation. The first problem was solved by proposing a hypothetical traction force in the longitudinal direction $[1,2]$ or by serially connecting an actuator to the spring-damper leg [13], so the energy dissipated by damping would be compensated. Solutions to the second problem mainly concern the transition of center of pressure (COP) under foot during the single-leg support phase. By adding a roller feet to the free end of spring leg or by directly modifying the contact point between foot and 
ground, Whittington and Thelen [14], Lim and Park [15], and Jung and Park [16], respectively, proposed their modified biped models (MBMs) that reduce the ankle bending angle, the COM motion amplitude, and the heel striking force at a relatively high walking speed.

The numerical studies based on these BMs and MBMs generally have the consensus to assume the leg stiffness as a whole instead of stiffness stemming from muscle tension and joints compliant bending. This idealization has made it possible to determine the effective leg stiffness according to the oscillatory natural frequency and inertial characteristics or according to Hooke's law which means dividing the magnitude of COM displacement by the GRF $[17,18]$. The optimal leg stiffness, which minimized the error between the model simulations and the measurements, would be obtained from a vast number of trials [16, 18, 19]. This compromise between the model simplification and the computation efforts has made it possible to predict the GRF with acceptable accuracy, but it also ignored the overall stiffness changes during the steps. The contribution of individual joint to the overall stiffness of the leg is not clear.

The calibration and validation of the model simulation should be based on a profound and robust empirical knowledge about the GRF data. Former research studies have qualitatively examined the shape and the symmetry of the single footfall profile [8] or quantitatively compared the magnitudes of double peaks [16]. These biomechanical databases were usually acquired from a small number of pedestrians and were presented and discussed within a single gait cycle. However, for other application purposes such as in the area of civil engineering, the knowledge about the inter- and intrasubject varieties was also necessary in predicting the loads induced by pedestrians to civil structures. As a result, a remarkable amount of GRF data from different subjects have been acquired using planar force plats [20-23]. Since the force-plate measurements should be implemented under well-prepared laboratory environments and a pair of force plates mounted on the floor or treadmill could only monitor and record a single footfall trace at a time, this kind of method was prone to the less practicality for quantifying the fluctuations during a real-life step-by-step walking [24]. The portable devices such as wireless inertial measurement units (IMUs) were supposed to be an alternative solution. This method has been proved to be able to predict the GRF indirectly according to the accelerations of body segments and also be able to record three dimensional components of accelerations [25].

This study aims at addressing the gap between experimental knowledge and model simulation by carrying out an observation of COM motion based on wearable wireless IMUs. We intended to regard the bending of knee joint as a controlled experimental condition. So the upper body accelerations were observed in natural walking and in fixedknee walking gait, respectively. Besides, considering the application in civil engineering, we transformed the time histories of COM accelerations into spectrum by fast Fourier transform and introduced the dynamic load factors (DLFs), which are the peaks of spectrum divided by the acceleration of gravity. Thus, the experimental data were available for model calibration and were compared with the computational results based on the PBM and the MBM simulations.

\section{Method}

2.1. Subjects. Six healthy youths (3 males and 3 females) were participated in the measurements. They were informed about the purpose and procedures of the research and were asked to sign an informed consent form prior to the tests. Their basic information is listed in Table 1 .

2.2. Protocols. Since the bending of knee joints was supposed to be the main control variable, the observations of the COM accelerations were implemented under two comparable conditions, i.e., the natural gait in which subjects could bend and stretch their knees freely (as shown in Figure 1(a)), and the fixed-knee gait in which bending of knee joints was prevented by the aid of orthosis depicted in Figure 1(b).

The subjects were asked to repeat several round trips at certain step frequencies $(1.6,1.8,2.0$, and $2.2 \mathrm{~Hz})$ on a straight walkway. The walkway was separated by marking lines into three consecutive sections (as shown in Figure 2)-a 3-meter-long access section for adjusting the gait from starting to the stable state, a $18 \mathrm{~m}$ observation section in which the stable gait information was monitored, and a $3 \mathrm{~m}$ exit section for turning around and restarting another trip.

Before the tests, the volunteers were asked to practice and maintain the steady-state walking under the guidance of a metronome and to get familiar with the walkway.

During the tests, one observer timed each trip with a smartphone-inbuilt stopwatch (which has a timing precision of $0.01 \mathrm{~s}$ ). By taking the heel striking as the indicator, the time of each trip was defined to begin with the first strike when stepping inside the observation section and to end up with the first strike when stepping out of this section (as shown in Figure 2). Another observer counted the number of steps with the same indicator. Under the condition that a subject walked with the step frequency of $2.0 \mathrm{~Hz}$ and with the stride length of $0.6 \mathrm{~m}$, the total time spent during each trip through the observation section would be about $15 \mathrm{~s}$. Assuming the maximum error due to observer for each trip is one step, the maximum timing error would be $0.5 \mathrm{~s}$ with respect to $15 \mathrm{~s}$, which equaled 0.033 .

The videos of leg behaviour were recorded by a smartphone-inbuilt camera fixed on a tripod, which is settled near the midpoint of the observation section (see Figure 2).

After the tests, we picked out the available part of acceleration signals corresponding to the observation section and calculated the walking speed and the stride length according to recorded time and step number.

2.3. Measurement. The COM accelerations were monitored and recorded by the wireless IMUs illustrated in Figure 3. The sensor is an integration of a three-axis accelerometer, a WiFi interface, and a power supply module. The acceleration signals are collected with a sampling rate of $50 \mathrm{~Hz}$, 
TABLE 1: Basic information of subjects.

\begin{tabular}{lcccc}
\hline No. & Gender & Age & Height $(\mathrm{cm})$ & Weight $(\mathrm{kg})$ \\
\hline 1 & Female & 25 & 168 & 174 \\
2 & Male & 24 & 173 & 69 \\
3 & Male & 27 & 160 & 59 \\
4 & Female & 25 & 162 & 47 \\
5 & Female & 26 & 180 & 62 \\
6 & Male & 29 & 66 \\
\hline
\end{tabular}

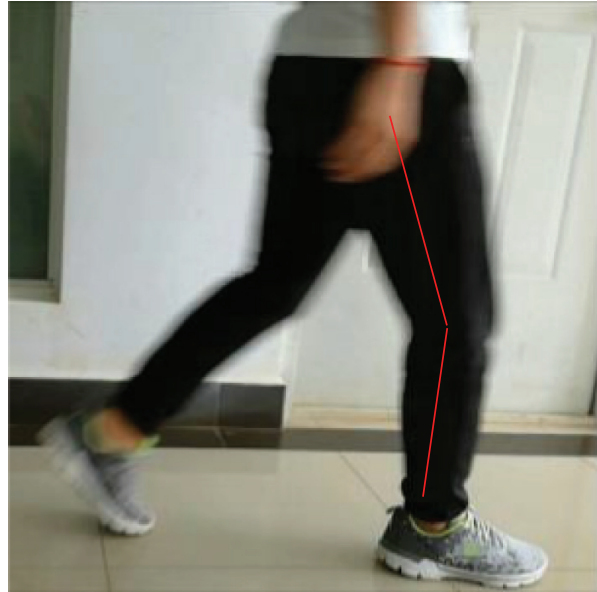

(a)

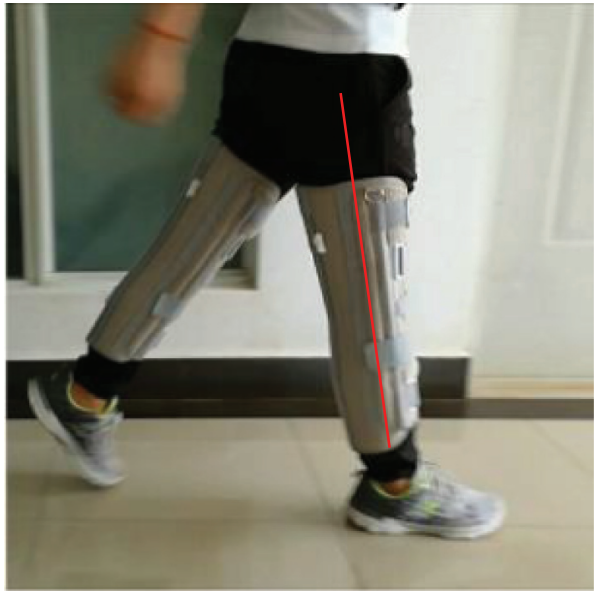

(b)

Figure 1: (a) Natural and (b) fixed-knee gait.

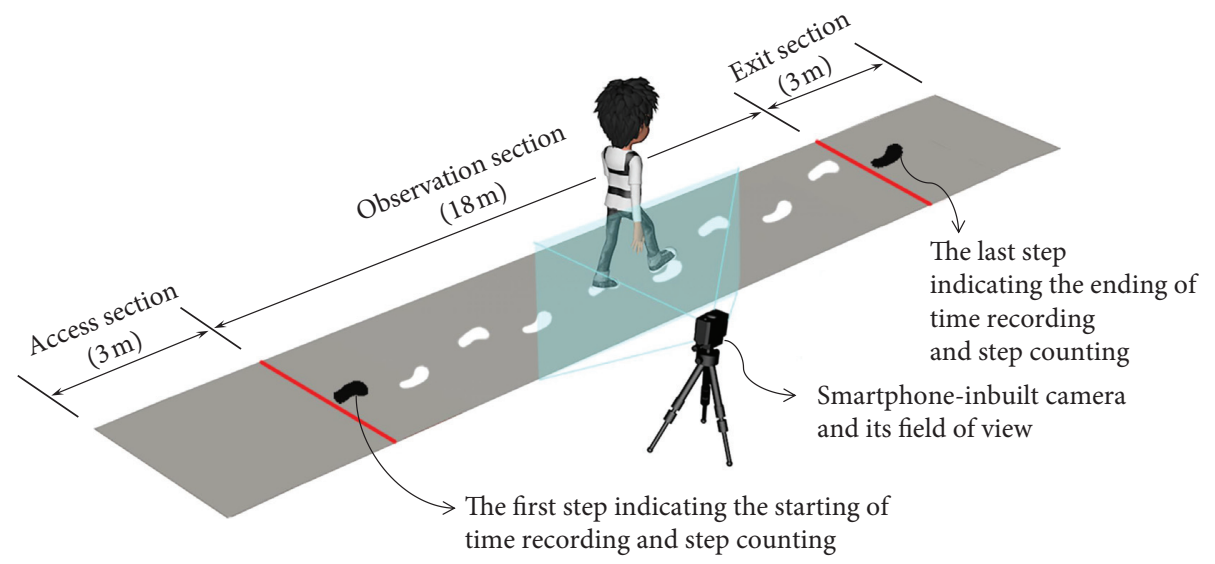

Figure 2: Schematic depiction (which was not drawn to scale) of the experimental settlement. During the tests, subjects repeated the round trips along the walkway. They were not informed about the separation of the walkway in case of any interference.

transmitted from the sensor to a host computer through a wireless network, and processed by low-pass filtering of $20 \mathrm{~Hz}$.

Two groups of sensors were arranged and fixed on the chest and the waist (as shown in Figure 3) to measure the accelerations of the upper and the lower torsos. Each group consisted of two sensors that were placed at the anterior and the posterior side of the body for the purpose of calibrating each other.
2.4. Biped Modal Simulation. A 3-degree-of-freedom model [2] examined in this article is shown in Figure 4. The upper and the lower torsos are represented by two lumped masses $m_{1}$ and $m_{2}$. The body and legs are simplified as springdamper units that have the original lengths of $l_{1}$ and $l_{2}$ and damping and stiffness coefficients of $c_{1}, c_{2}, k_{1}$, and $k_{2}$. The angles of the leading and trilling legs to the rigid surface $\theta_{l}$ and $\theta_{t}$ vary during walking. The motion equation of the above system is as follows [2]: 


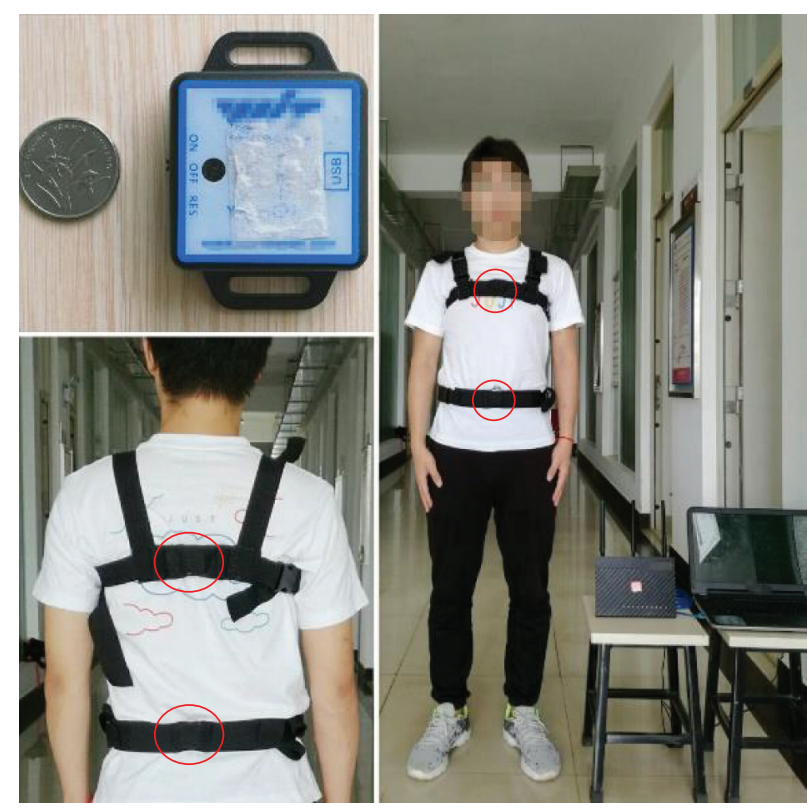

Figure 3: Wireless sensors and layout.

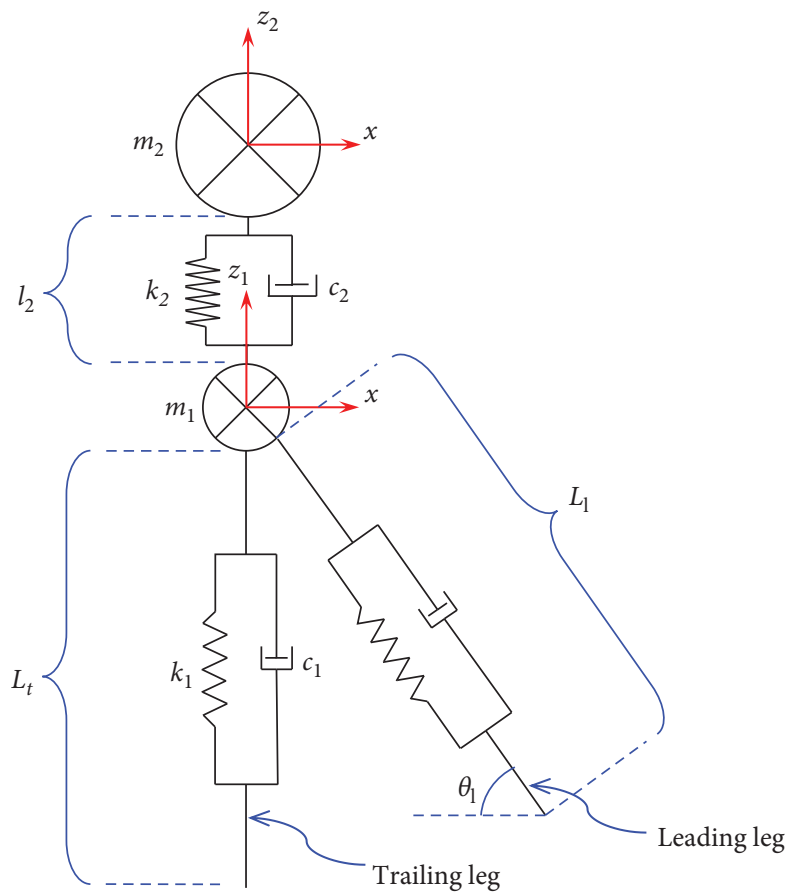

Figure 4: Primitive biped model with two lumped COMs. Note that $m_{2}$ is always above $m_{1}$ during motion. Torques introduced by the legs about $m_{2}$ are ignored. 


$$
M \ddot{X}+C \dot{X}+K X=F,
$$

in which $\mathbf{X}$ represents the displacement vectors, including the vertical displacements of upper and lower COMs $z_{1}$ and $z_{2}$ and the longitudinal displacement $x$ of the whole body:

$$
X^{T}=\left[\begin{array}{lll}
z_{1} & z_{2} & x
\end{array}\right] .
$$

$M, C, K$, and $F$ are mass, damping, stiffness, and force matrices:

$$
\begin{aligned}
& M=\left[\begin{array}{ccc}
m_{1} & 0 & 0 \\
0 & m_{2} & 0 \\
0 & 0 & m_{1}+m_{2}
\end{array}\right] \\
& C=\left[\begin{array}{ccc}
c_{l} \sin ^{2} \theta_{l}+c_{t} \sin ^{2} \theta_{t}+c_{2} & -c_{2} & -c_{l} \sin \theta_{l} \cos \theta_{l}+c_{t} \sin \theta_{t} \cos \theta_{t} \\
-c_{2} & c_{2} & 0 \\
-c_{l} \sin \theta_{l} \cos \theta_{l}+c_{t} \sin \theta_{t} \cos \theta_{t} & 0 & c_{l} \cos ^{2} \theta_{l}+c_{t} \cos ^{2} \theta_{t}
\end{array}\right] \text {, } \\
& K=\left[\begin{array}{ccc}
k_{l, v}+k_{t, v}+k_{2} & -k_{2} & 0 \\
-k_{2} & k_{2} & 0 \\
0 & 0 & -k_{l, h}+k_{t, h}
\end{array}\right] \text {, } \\
& F=\left[\begin{array}{c}
-m_{1} g-k_{2} l_{2} \\
-m_{2} g+k_{2} l_{2} \\
F_{c t r l}
\end{array}\right] \text {. }
\end{aligned}
$$

A longitudinal control force [1] is introduced to compensate for the energy dissipated by the damping of torsos and legs, ensuring a long-term steady-state walking. It is expressed as

$$
\begin{aligned}
F_{c t r l}= & \frac{E_{0}-E(t)}{\Delta x(t)}, \\
E(t)= & \frac{1}{2} m_{1} \dot{z}_{1}^{2}+\frac{1}{2} m_{2} \dot{z}_{2}^{2}+\frac{1}{2}\left(m_{1}+m_{2}\right) \dot{x}^{2} \\
& +\frac{1}{2} k_{1}\left(l_{1}-L_{l}\right)^{2}+\frac{1}{2} k_{1}\left(l_{1}-L_{t}\right)^{2}+\frac{1}{2} k_{2}\left(z_{2}-z_{1}-l_{2}\right)^{2}, \\
& +m_{1} g z_{1}+m_{2} g z_{2}
\end{aligned}
$$

in which $E$ is the total kinetic and potential energy of the system and $L_{l}$ and $L_{t}$ represent the actual length of the leading and the trilling legs during walking, which are calculated according to the step length $d$ and the longitudinal displacement of COM shown in the following equation:

$$
\left\{\begin{array}{l}
L_{l}=\sqrt{\left(\sum_{i=1}^{N} d_{i}-x\right)^{2}+z_{1}^{2}}, \\
L_{t}=\sqrt{z_{1}^{2}+\left(x-\sum_{i=1}^{N-1} d_{i}\right)^{2}} .
\end{array}\right.
$$

The elements of stiffness and damping matrices alternate according to the single or the double support phase. As for the double support phase, their expressions are

$$
\left\{\begin{aligned}
k_{l, v} & =k_{1}\left(1-\frac{l_{1}}{L_{l}}\right), \\
k_{t, v} & =k_{1}\left(1-\frac{l_{1}}{L_{t}}\right), \\
k_{l, h} & =k_{1}\left(1-\frac{l_{1}}{L_{l}}\right)\left(\sum_{i=1}^{N} \frac{d_{i}}{x}-1\right), \\
k_{t, h} & =k_{1}\left(1-\frac{l_{1}}{L_{t}}\right)\left(1-\sum_{i=1}^{N-1} \frac{d_{i}}{x}\right), \\
c_{l} & =\alpha(t) c_{1}, \\
c_{t} & =[1-\alpha(t)] c_{1}, \\
\alpha(t) & =\frac{L_{t}(t)-L_{t}(0)}{l_{1}-L_{t}(0)}
\end{aligned}\right.
$$

where the ratio $\alpha$ indicates that the total damping is distributed to the leading and the trilling leg. It is determined by the actual length of the trilling leg. As for the single support 
phase, we assume that the trilling leg is swaying without the changes in length, which keeps the original length $L_{t}=l_{1}$. The elements in Equations (5) and (6) should be updated accordingly.

A modified biped model [16] suggests an empirical expression of COP excursion from the heel to toe-plantar joint, which is given as

$$
\begin{aligned}
x_{c o p} & =-\frac{2 d_{f}}{3 \pi}\left(\sin \omega t-\frac{1}{8} \sin 2 \omega t-\frac{3}{4} \omega t\right), \\
\omega & =\gamma \omega_{n}=\gamma \sqrt{\frac{k_{1}}{m_{1}+m_{2}}},
\end{aligned}
$$

where $d_{f}$ is the length of sole that has an average value of $15 \mathrm{~cm}$. $\omega$ is defined as $2 \pi / T_{s s}$, where $T_{s s}$ is the time period of single support phase. $\omega$ is assumed to be proportional to the natural frequency $\omega_{n}$ of model, and the ratio $\gamma$ takes the value of 1 . Because step length is determined by the distance between COP of two legs, above suggestion for COP excursion leads to the change in step length during the single support phase:

$$
d_{i}^{\prime}=d_{i}+x_{c o p}
$$

Accordingly, length and stiffness of supporting leg would become

$$
\begin{gathered}
L_{i}^{\prime}=\sqrt{\left(\sum_{i=1}^{N} d_{i}^{\prime}-x\right)^{2}+z_{1}^{2},} \\
\left\{\begin{array}{l}
k_{l, v}^{\prime}=k_{1}\left(1-\frac{l_{1}}{L_{l}^{\prime}}\right), \\
k_{l, h}^{\prime}=k_{1}\left(1-\frac{l_{1}}{L_{l}^{\prime}}\right)\left(\sum_{i=1}^{N} \frac{d_{i}^{\prime}}{x}-1\right) .
\end{array}\right.
\end{gathered}
$$

The motion equations for MBM would be acquired by replacing Equations (5) and (6) of PBM with Equations (12) and (13).

Simulations based on above motion equations are implemented under the parameter settings summarized in Table 2. The anthropometric data such as leg and torso lengths in Table 2 were determined by the height of waist and the relative height between chest and waist under the natural standing position. Total weight was divided into upper and lower parts according to the ratio of $m_{1} / m_{2}=0.17$ that was suggested by ISO 5982 [26]. The leg stiffness was picked out from the range of $12-20 \mathrm{kN} / \mathrm{m}$ proposed in $[1,2]$ to make sure the simulated step frequency $f_{p}$ was conformed with measured step frequency. Torso stiffness $k_{2}$, leg damping ratio $\xi_{1}$, and torso damping coefficient $c_{2}$ took the certain values referring to [2].

The Newton-Raphson iteration with a time step of $0.001 \mathrm{~s}$ was applied to solve motion equations and acquire a 40-second stable walking for each trip.
2.5. Dynamic Load Factors. The accelerations of COM acquired from simulation and/or measurement can be transformed into the expression of Fourier series to acquire dynamic load factors (DLFs) [20], as shown in the following equations:

$$
\begin{aligned}
& \ddot{z}=\frac{1}{2}\left(\ddot{z}_{1}+\ddot{z}_{2}\right)=g+g \sum_{i=1}^{M}\left[D L F_{z i} \sin \left(2 i \pi f_{p} t-\varphi_{z i}\right)\right] \\
& \ddot{x}=g \sum_{i=1}^{M}\left[D L F_{x i} \sin \left(2 i \pi f_{p} t-\varphi_{x i}\right)\right]
\end{aligned}
$$

where $g$ is the acceleration due to gravity, $D L F_{z i}$ and $D L F_{x i}$ represent the $i^{\text {th }}$ order of vertical and longitudinal dynamic load factors, $\varphi_{z i}$ and $\varphi_{x i}$ are the harmonic phase angles of relevant direction, and $f_{p}$ is the step frequency.

\section{Results}

3.1. Acceleration Records. The accelerations corresponding to steady-state walking are picked out from the truncation of the original signals. The specific procedures are illustrated in Figure 5, in which the vertical accelerations of volunteer No. 1 are taken as examples. Figure 5(a) records the vertical accelerations acquired by the sensor at the chest during the whole measurement under natural walking. It shows an increasing trend of the amplitudes of time histories with the increase in step frequencies from $1.6 \mathrm{~Hz}$ to $2.2 \mathrm{~Hz}$. In a smaller scale shown in Figure 5(b), the accelerations under a specific step frequency (i.e., $2.0 \mathrm{~Hz}$ ) consist of two phases: the walking phases that generally have higher amplitudes and the turning round phases with lower amplitudes. The details within each walking phase are displayed in Figure 5(c), in which the acceleration ascends and descends, respectively, at the beginning and the ending of the period, indicating the volunteer is accelerating and decelerating, while the stable acceleration in the intermediate period illustrates a steadystate walking.

Typical time histories of measured and simulated accelerations of volunteer No. 1 at the step frequency of $2.0 \mathrm{~Hz}$ are plotted and compared in Figure 6 . Figure 6 illustrates the accelerations in vertical $(\overline{\ddot{z}})$ and longitudinal $(\overline{\ddot{x}})$ directions. The legends EC and EB represent the experimental results in the condition of the natural and the fixed-knee gait, respectively, while PBM and MBM represent the numerical results of the PBM and the MBM, respectively. It is noted that the amplitudes of numerical curves are larger than those of experimental curves.

The time histories of steady-state accelerations are transformed into frequency spectra trough fast Fourier transform (FFT). Figure 7 shows the typical vertical and longitudinal spectra of volunteer No. 1 walking under the step frequency of $2.0 \mathrm{~Hz}$. To compare the simulated spectra with the measured spectra, the peaks of the first order of harmonic are summarized in Table $4 \mathrm{We}$ can infer from 
TABLE 2: Length and mass of body.

\begin{tabular}{lcccc}
\hline No. & $l_{1}(\mathrm{~cm})$ & $l_{2}(\mathrm{~cm})$ & $m_{1}(\mathrm{~kg})$ & \\
\hline 1 & 90 & 40 & 10.9 & 52 \\
2 & 93 & 41 & 12 & 57 \\
3 & 93.5 & 42.5 & 10.2 & 48.8 \\
4 & 83.5 & 39.5 & 8.1 & 38.9 \\
5 & 83 & 41 & 10.7 & 51.3 \\
6 & 101 & 41 & 11.4 & 54.6 \\
\hline
\end{tabular}

TABLe 3: Stiffness and damping.

\begin{tabular}{lcccc}
\hline$f_{p}(\mathrm{~Hz})$ & $k_{1}(\mathrm{kN} / \mathrm{m})$ & $k_{2}(\mathrm{kN} / \mathrm{m})$ & $\xi_{1}$ & \\
1.8 & 14 & & & \\
2.0 & 17 & 62 & 0.08 & 14.6 \\
2.2 & 20 & & & \\
\hline
\end{tabular}
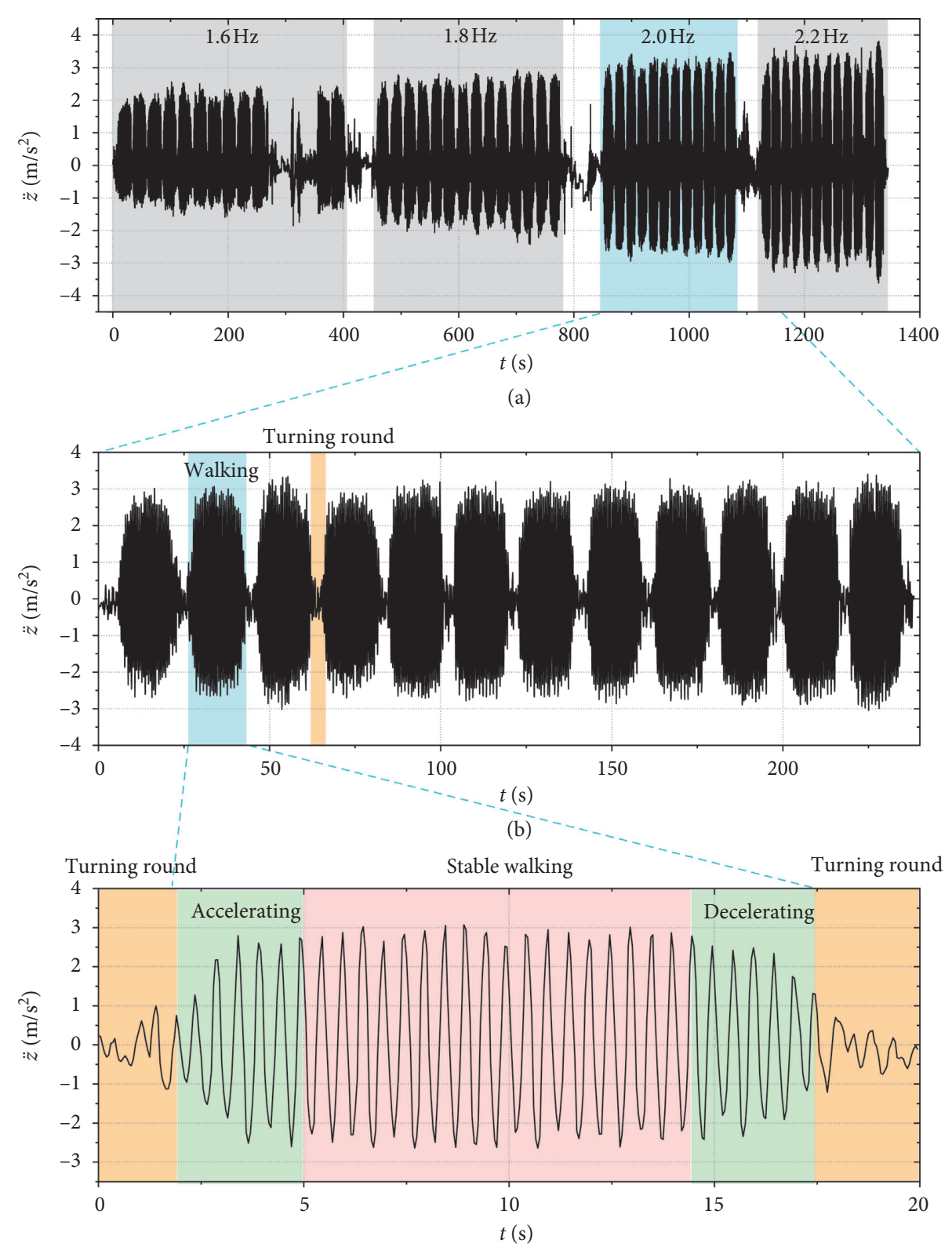

(c)

Figure 5: : Signals acquired by the sensor at chest of volunteer No. 1 in natural walking. During the whole test shown in (a), peaks of accelerations increase with the step frequencies. Within the time walking in $2.0 \mathrm{~Hz}$ shown in (b), accelerations during walking are different from that during turning round. For each walking duration shown in (c), we only analyze the stable parts (in the red area), ignoring unstable acceleration signals (in the blue area). 


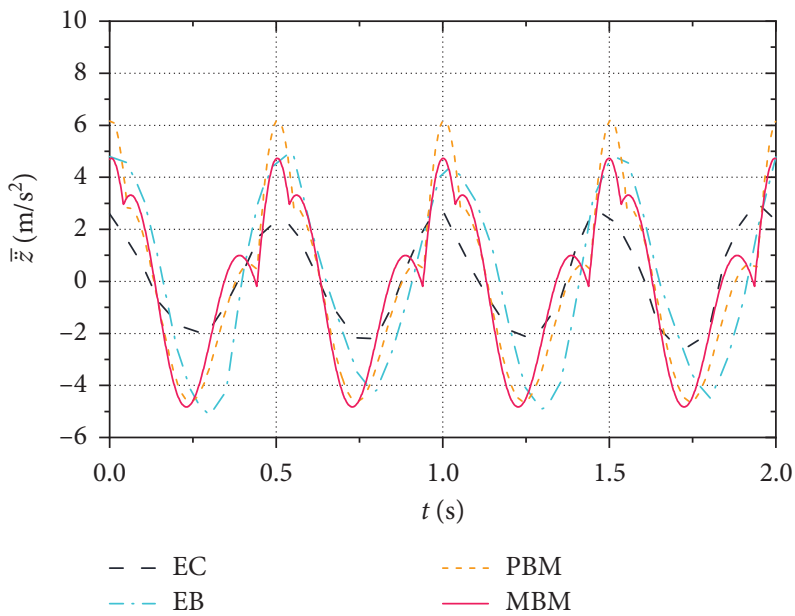

(a)

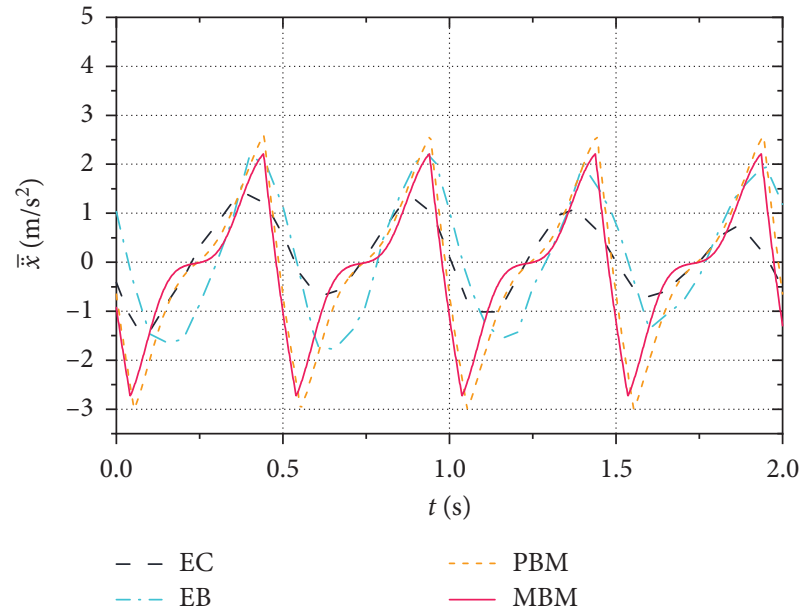

(b)

FIgURE 6: COM accelerations of volunteer No. 1 walking at the step frequency of $2.0 \mathrm{~Hz}$ in (a) vertical and (b) longitudinal directions.

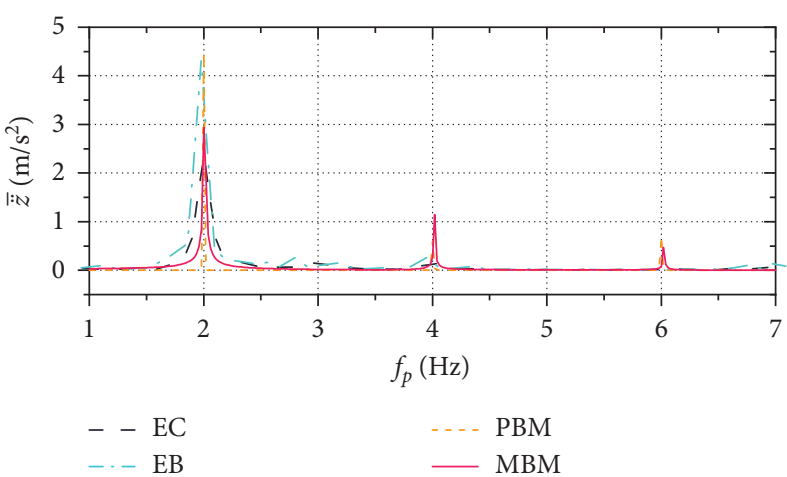

(a)

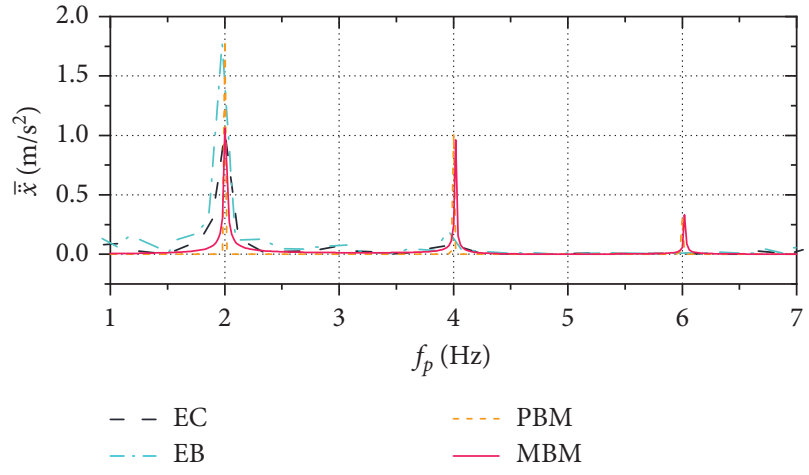

(b)

FiguRE 7: Frequency spectra of accelerations of volunteer No. 1 walking under the step frequency of $2.0 \mathrm{~Hz}$.

TABle 4: Peak values of the first-order harmonics of volunteer No. 1 under the step frequency of $2.0 \mathrm{~Hz}$.

\begin{tabular}{lcccc}
\hline Directions & Results & Sample numbers & Average values $\left(\mathrm{m} / \mathrm{s}^{2}\right)$ & Ranges $\left(\mathrm{m} / \mathrm{s}^{2}\right)$ \\
\hline & EC & 10 & 2.50 & $2.27-2.77$ \\
$\mathrm{Z}$ & EB & 6 & 3.81 & $3.52-4.12$ \\
& PBM & 1 & 4.48 & $/$ \\
& MBM & 1 & 3.54 & 1 \\
$\mathrm{X}$ & EC & 10 & 1.19 & $1.13-1.32$ \\
& EB & 6 & 1.81 & $1.68-1.94$ \\
& PBM & 1 & 1.81 & $/$ \\
\hline
\end{tabular}

Table 4 the following: the PBM results are the largest among the four kinds of results, while the MBM results are larger than the natural walking results and are smaller than the fixed-knee walking results.

3.2. Dynamic Load Factors. The DLFs are derived from peak values of spectra according to their definitions expressed by Equations (14) and (15). The first and the second orders of
DLFs are displayed in this paper (see Figures 8 and 9), which contain the measured and the simulated DLFs of six volunteers. The sample points in Figure 8 or Figure 9 consist of 240 samples of natural walking, 144 samples of fixed-knee walking, and 18 samples of each kind of BMs.

Based on the layout of the sample points, it is reasonable to assume that DLFs have a linear relationship with step frequency, which is regressed and expressed as 


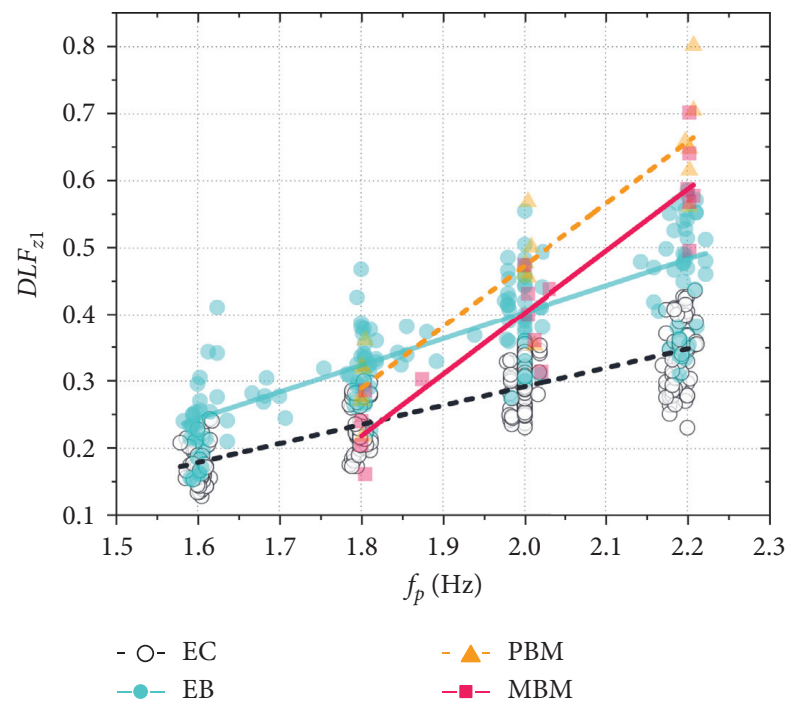

(a)

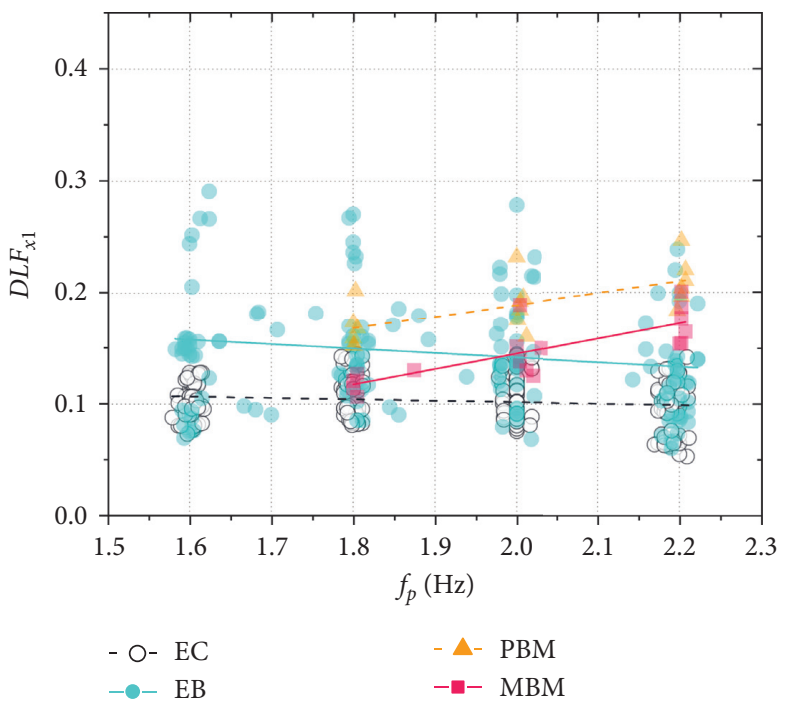

(b)

FIgURE 8: DLFs of the first order in (a) vertical and (b) longitudinal directions.

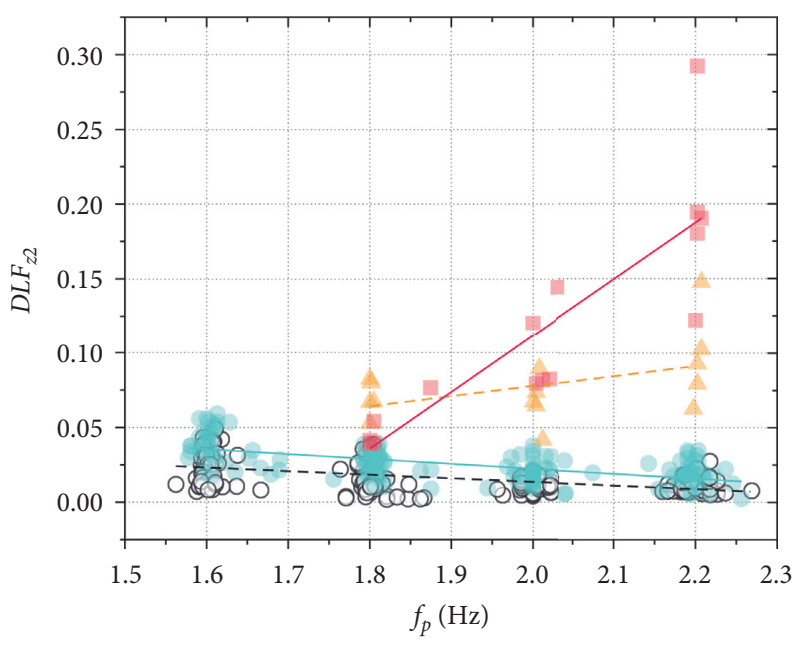

- O- EC

$-\bullet-E C$
$\triangle-\mathrm{PBM}$

$-\mathrm{MBM}$

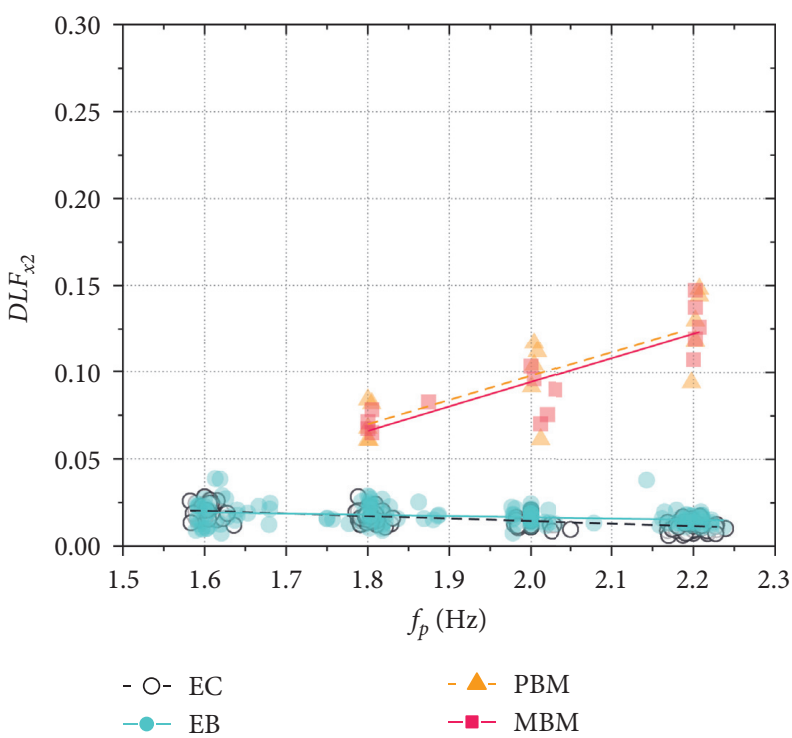

(b)

FIgURE 9: DLFs of the second order in (a) vertical and (b) longitudinal directions.

$$
\left\{\begin{array}{l}
D L F_{z i}=\beta_{z i}\left(a_{z i} f_{p}+b_{z i}\right), \\
D L F_{x i}=\beta_{x i}\left(a_{x i} f_{p}+b_{x i}\right),
\end{array}\right.
$$

where parameters $a_{z i}, a_{x i}, b_{z i}$, and $b_{x i}$ get the fitting values, as shown in Table $5 ; \beta_{z i}$ and $\beta_{x i}$ are defined as coefficient of variation for describing the deviations of measured values from fitting results and are prone to subject to normal distribution, as shown in Figures 10 and 11.

\section{Discussion}

This study attempts to investigate the accuracy of BM to anticipate acceleration of COM by a single-leg stiffness. The effects of the compliance in ankle, foot, and knee joints are examined by comparing the BM simulation results with those measured through wireless acceleration sensors.

On the one hand, to evaluate the effects of ankle and foot compliance, we compared the PBM simulations that ignore the foot and ankle joint with the MBM simulations that 
TABLE 5: Linear fittings of total DLFs.

\begin{tabular}{lccc}
\hline Sample & $a_{z 1}$ & $b_{z 1}$ & RSQ \\
\hline EC & 0.2834 & -0.2746 & 0.73 \\
EB & 0.3981 & -0.3931 & 0.67 \\
PBM & 0.9215 & -1.3696 & 0.85 \\
MBM & 0.9219 & -1.4415 & 0.87 \\
& $a_{x 1}$ & $b_{x 1}$ & RSQ \\
EC & -0.0123 & 0.1265 & 0.02 \\
EB & -0.0397 & 0.2212 & 0.03 \\
PBM & 0.1052 & -0.0213 & 0.43 \\
MBM & 0.1380 & -0.1307 & 0.62 \\
& $a_{z 2}$ & $b_{z 2}$ & RSQ \\
EC & -0.0239 & 0.0614 & 0.26 \\
EB & -0.0327 & 0.0877 & 0.36 \\
PBM & 0.0680 & -0.0583 & 0.21 \\
MBM & 0.3790 & -0.6465 & 0.73 \\
& $a_{x 2}$ & $b_{x 2}$ & RSQ \\
EC & -0.0143 & 0.0431 & 0.51 \\
EB & -0.0069 & 0.0304 & 0.07 \\
PBM & 0.1392 & -0.1807 & 0.64 \\
MBM & 0.1405 & -0.1869 & 0.74 \\
\hline
\end{tabular}

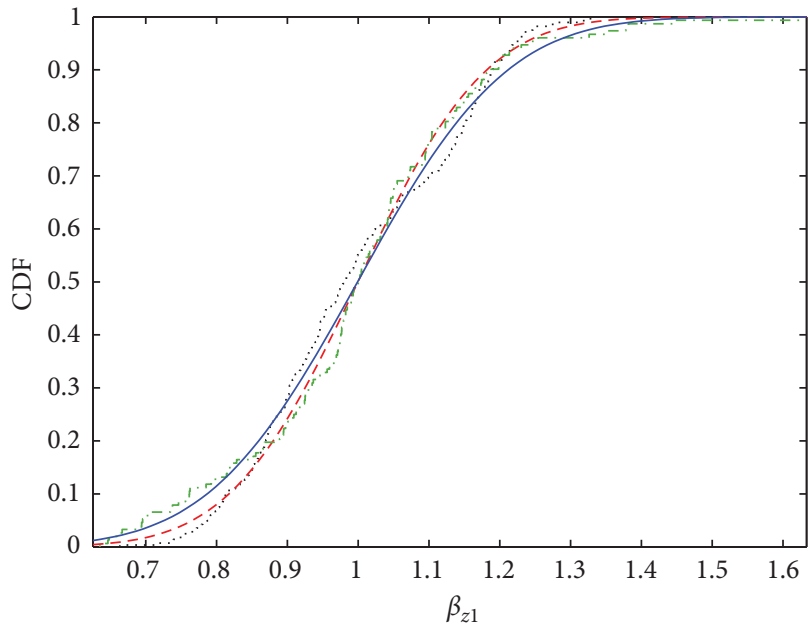

$$
\begin{array}{ll}
\cdots & \text { EC } \\
\cdots & N(\mu=1, \sigma=0.14) \quad-N(\mu=1, \sigma=0.17)
\end{array}
$$

(a)

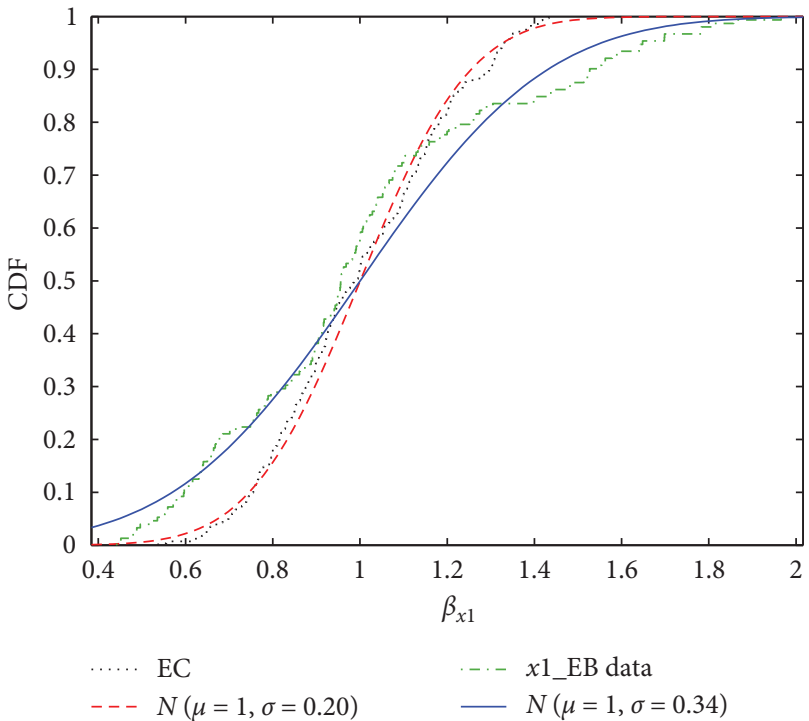

(b)

Figure 10: Cumulative density function (CDF) of variation coefficient of the first-order DLF in (a) vertical and (b) longitudinal directions.

considered the excursion of COP due to the ankle and foot compliance. According to the time histories and frequency spectra of COM accelerations shown in Figure 6, Figure 7, and Table 4, the results of MBM have less errors than those of PBM comparing the natural walking measurements, revealing that taking into account the compliance of ankle and foot improves the accuracy of biped model. This evidence further supports the conclusion of former studies [16, 19] that consideration of the COP excursion enables a more accurate prediction of the single step force within a wider range of walking speed.

On the other hand, to evaluate the influence of compliant knee bending, the COM accelerations under the natural and fixed-knee gait were, respectively, measured and were compared with simulation results. According to the first order of DLFs depicted in Figure 8 and Equation (14), the discrepancies between BM simulations and measured results depend on the step frequencies. Under the condition that the step frequencies are lower than $2.0 \mathrm{~Hz}, \mathrm{MBM}$ results lie between natural and fixed-knee walking results and have less discrepancy to both of them. If the step frequencies are higher than $2.0 \mathrm{~Hz}, \mathrm{MBM}$ results exceed natural walking results for $60 \%$ while exceed the average of fixed-knee walking results for less than $20 \%$. These results indicate that the COM accelerations simulated by MBM have remarkable increasing discrepancy to those of natural gait under a 


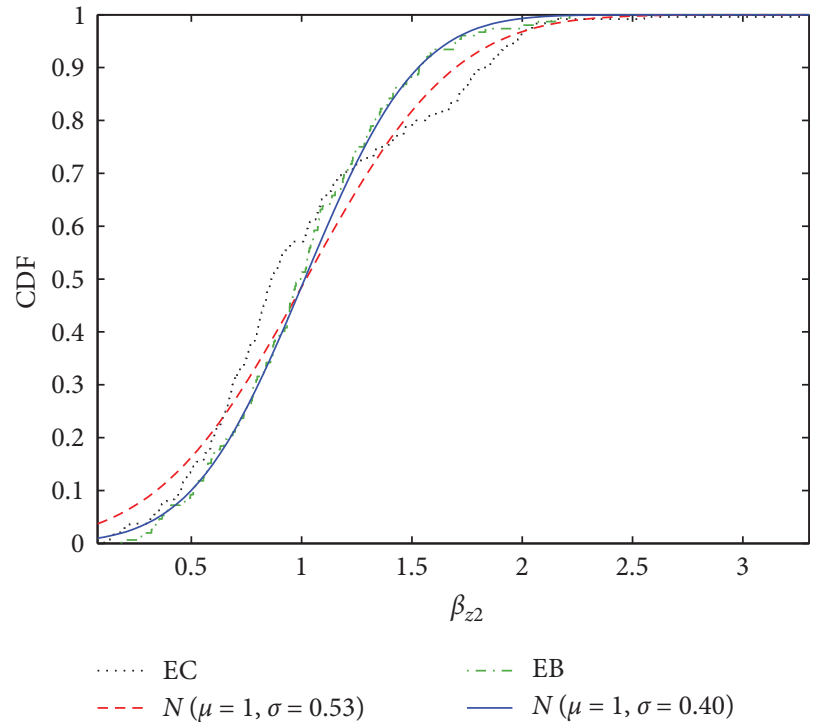

(a)

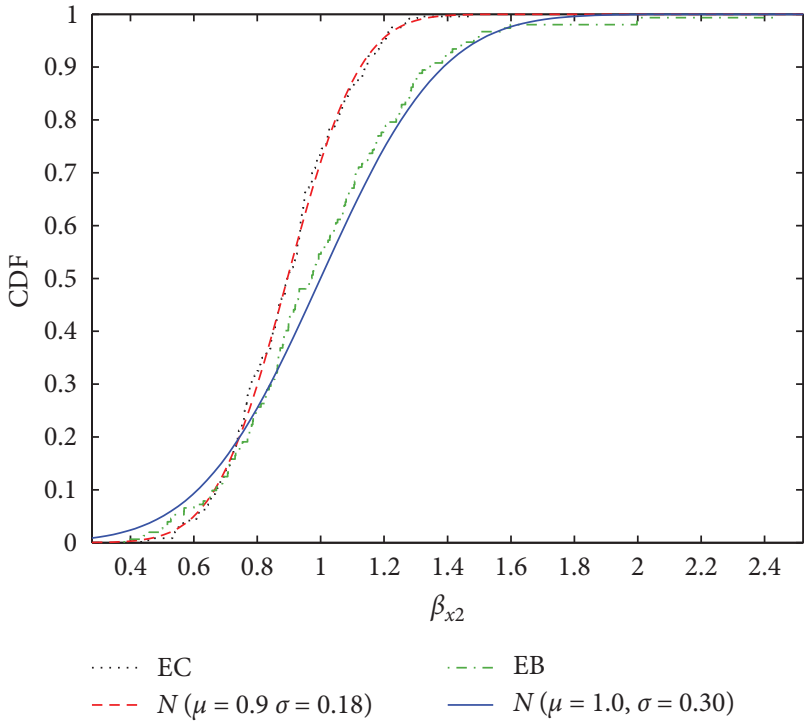

(b)

FIgURE 11: Cumulative density function (CDF) of variation coefficient of the second-order DLF in (a) vertical and (b) longitudinal directions.

TABLE 6: DLF of former force-plate measurements.

\begin{tabular}{|c|c|c|}
\hline Researchers & DLF & Statistics \\
\hline \multirow{2}{*}{ Chen [20] } & $\begin{array}{c}D L F_{z 1}=0.2358 f_{p}-0.2611 \\
D L F_{z 2}=0.0757\end{array}$ & Mean \\
\hline & $\begin{array}{c}D L F_{z 1}=0.2358 f_{p}-0.2010 \\
D L F_{z 2}=0.0949\end{array}$ & $75 \%$ fractile \\
\hline Kerr [21] and Zivanovic [22] & $\begin{array}{c}D L F_{z 1}=-0.2649 f_{p}^{3}+1.3206 f_{p}^{2}-1.7597 f_{p}+0.7613 \\
D L F_{z 2}=0.07\end{array}$ & Mean \\
\hline Young [23] & $\begin{array}{c}D L F_{z 1}=0.41 f_{p}-0.3895 \\
D L F_{z 2}=0.0056 f_{p}+0.069\end{array}$ & Mean \\
\hline
\end{tabular}

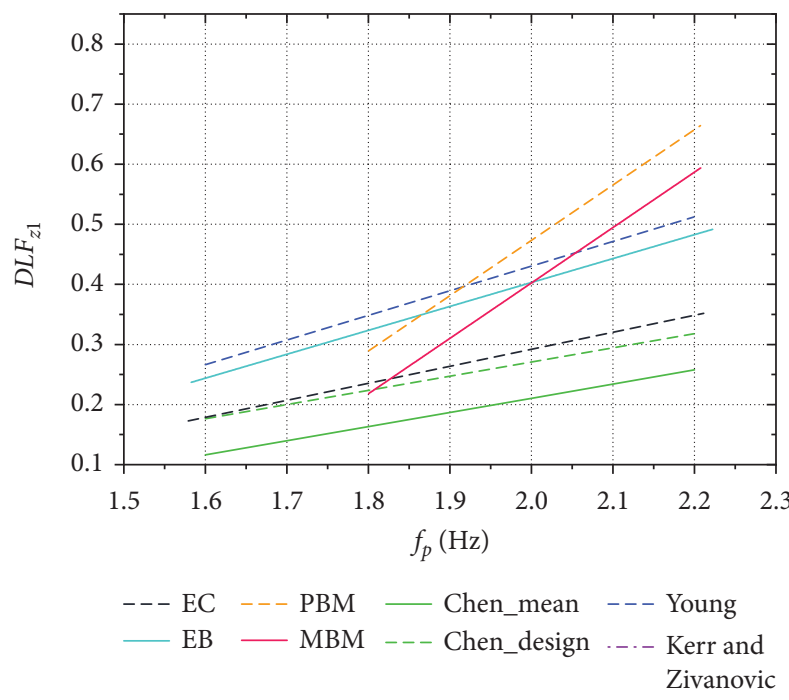

(a)

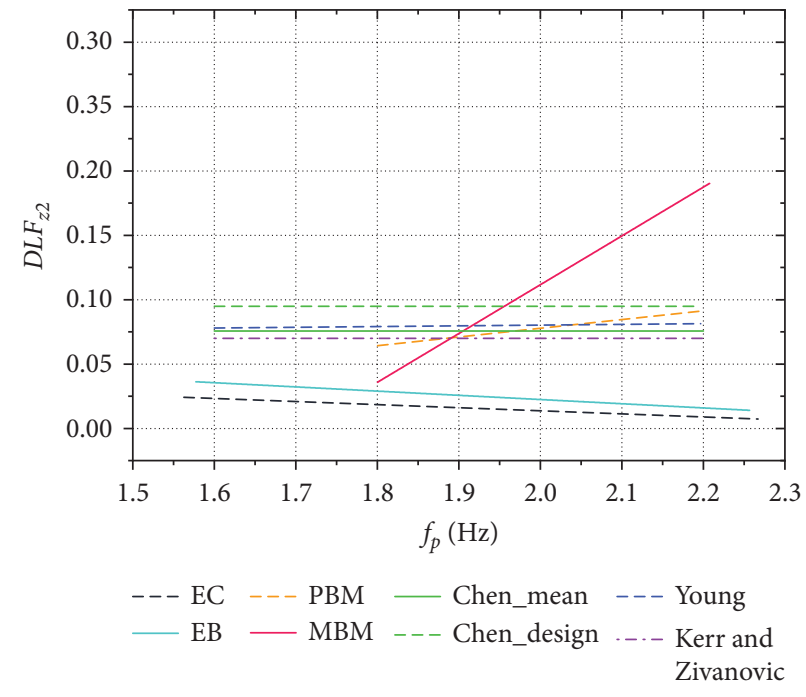

(b)

FIGURE 12: DLFs of (a) the first and (b) the second orders in the vertical direction. 
higher step frequency. These results are in conform with observations in former studies $[16,18,19]$, which also show that model predictions have higher deviation from measurements in terms of peaks of single step force under higher walking speed (namely, the higher step frequency).

The DLFs acquired through COM acceleration measurement were compared with those proposed in former studies [20-23] based on force-plate measurement. Table 6 shows corresponding expressions of fitting results. Figure 12 contains only the results of vertical DLFs because the force plate cannot measure the longitudinal forces directly. It is noted that similar results are derived from different measurement methods. As for the first order of DLFs in Figure 12(a), the linear relationship between DLFs and step frequency from different methods has a similar slope, even though our results are closer to those based on Chinese volunteers than those based on European samples (considering the variance of body geometrical features). As for the second order of DLFs in Figure 12(b), both methods indicate a weak relationship between DLFs and step frequency. Our results are relatively low due to the lower sampling rate of wireless sensors. It suggests that further experimental research studies should seek a trade-off between the accuracy and the minimum level of interruption to subjects.

Comparing with the fitting results of above methods, those of PBM and MBM show larger slopes (especially for the second order of MBM results in Figure 12(b)). The discrepancy indicates that the mechanism of knee bending should be further investigated, and corresponding modifications should be implemented to present biped models before they may accurately predict the COM accelerations.

\section{Conclusions}

We compared the COM accelerations simulated by two kinds of BMs with those measured under natural walking and fixed-knee walking in terms of DLFs. The results indicate the following:

(1) The first order of DLFs predicted by PBM is 37\% lower than those of MBM simulation. This difference in levels could be attributed to the consideration of the transition of the COP under feet and the compliant bending of ankle joints during walking.

(2) The first order of DLFs measured under fixed-knee walking have at most $66 \%$ higher levels than those under natural walking. This difference could result from limiting the bending of knee.

(3) Though the measurements of fixed-knee walking are closer to MBM simulations than the measurements under natural walking, the discrepancy between measurements and simulations could not only be attributed to the bending of knee. The results of fixed-knee cannot explain the different slopes of DLFs with respect to step frequencies. The errors of $\mathrm{BM}$ to anticipate realistic COM accelerations may be a result of multiple reasons such as wrist, knee, and ankle compliance rather than a single aspect. Further research studies are expected to investigate the mechanisms.

(4) In engineering applications, such as the vibration serviceability design of structural engineering, the walking loads anticipated by BMs are acceptable within the step frequencies less than $2.0 \mathrm{~Hz}$. Under the higher frequencies, the error could be $60 \%$ at most. One should be cautious about BM simulation results under these conditions, and reasonable adjustments should be considered.

\section{Data Availability}

The text data of acceleration signals used to support the findings of this study are available from the corresponding author upon request.

\section{Conflicts of Interest}

The authors declare that there are no conflicts of interest regarding the publication of this paper.

\section{Acknowledgments}

The authors acknowledge the financial support which came from the National Natural Science Foundation of China (Grant no. 51168021) and the Science Foundation of Yunnan Provincial Department of Education (Grant no. 2017ZZX187).

\section{References}

[1] J. W. Qin, S. S. Law, Q. S. Yang et al., "Smart modeling of the human-structure interaction with human intervention," Dynamics for Sustainable Engineering, vol. 4, pp. 1757-1766, 2011.

[2] Y. Wang and N. Yang, "Walking loads analysis based on a new bipedal model and its parameters effect," Journal of Vibration and Shock, vol. 34, no. 13, pp. 152-157, 2015.

[3] Z. Sun and N. Roos, "Dynamically stable walk control of biped humanoid on uneven and inclined terrain," Neurocomputing, vol. 280, pp. 111-122, 2018.

[4] T. F. Besier, M. Fredericson, G. E. Gold, G. S. Beaupré, and S. L. Delp, "Knee muscle forces during walking and running in patellofemoral pain patients and pain-free controls," Journal of Biomechanics, vol. 42, no. 7, pp. 898-905, 2009.

[5] H. M. Herr and A. M. Grabowski, "Bionic ankle-foot prosthesis normalizes walking gait for persons with leg amputation," Proceedings of the Royal Society B: Biological Sciences, vol. 279, no. 1728, pp. 457-464, 2012.

[6] E. Shahabpoor, A. Pavic, and V. Racic, "Interaction between walking humans and structures in vertical direction: a literature review," Shock and Vibration, vol. 2016, Article ID 3430285, 22 pages, 2016.

[7] J. Sim, A. Blakeborough, and M. Williams, "Modelling of joint crowd-structure system using equivalent reduced-DOF system," Shock and Vibration, vol. 14, no. 4, pp. 261-270, 2007.

[8] H. Geyer, A. Seyfarth, and R. Blickhan, "Compliant leg behaviour explains basic dynamics of walking and running," Proceedings of the Royal Society B: Biological Sciences, vol. 273, no. 1603, pp. 2861-2867, 2006. 
[9] M. Srinivasan and A. Ruina, "Computer optimization of a minimal biped model discovers walking and running," $\mathrm{Na}$ ture, vol. 439, no. 7072, pp. 72-75, 2006.

[10] G. A. Cavagna, H. Thys, and A. Zamboni, "The sources of external work in level walking and running," The Journal of Physiology, vol. 262, no. 3, pp. 639-657, 1976.

[11] H. Hong, S. Kim, C. Kim, S. Lee, and S. Park, "Spring-like gait mechanics observed during walking in both young and older adults," Journal of Biomechanics, vol. 46, no. 1, pp. 77-82, 2013.

[12] M. Lee, S. Kim, and S. Park, "Resonance-based oscillations could describe human gait mechanics under various loading conditions," Journal of Biomechanics, vol. 47, no. 1, pp. 319-322, 2014.

[13] T. Li, Q. Li, and T. Liu, "An actuated dissipative spring-mass walking model: predicting human-like ground reaction forces and the effects of model parameters," Journal of Biomechanics, vol. 90, pp. 58-64, 2019.

[14] B. R. Whittington and D. G. Thelen, "A simple mass-spring model with roller feet can induce the ground reactions observed in human walking," Journal of Biomechanical Engineering - Transactions of the Asme, vol. 131, no. 1, 2009.

[15] H. Lim and S. Park, "Kinematics of lower limbs during walking are emulated by springy walking model with a compliantly connected, off-centered curvy foot," Journal of Biomechanics, vol. 71, pp. 119-126, 2018.

[16] C. K. Jung and S. Park, "Compliant bipedal model with the center of pressure excursion associated with oscillatory behavior of the center of mass reproduces the human gait dynamics," Journal of Biomechanics, vol. 47, no. 1 , pp. 223-229, 2014.

[17] D. P. Ferris, M. Louie, and C. T. Farley, "Running in the real world: adjusting leg stiffness for different surfaces," Proceedings of the Royal Society of London. Series B: Biological Sciences, vol. 265, no. 1400, pp. 989-994, 1998.

[18] S. Kim and S. Park, "Leg stiffness increases with speed to modulate gait frequency and propulsion energy," Journal of Biomechanics, vol. 44, no. 7, pp. 1253-1258, 2011.

[19] H. X. Ryu and S. Park, "Estimation of unmeasured ground reaction force data based on the oscillatory characteristics of the center of mass during human walking," Journal of Biomechanics, vol. 71, pp. 135-143, 2018.

[20] J. Chen, H. Wang, and Y. Peng, "Experimental investigation on Fourier-series model of walking load and its coefficients," Journal of Vibration and Shock, vol. 33, no. 8, pp. 11-15, 2014.

[21] S. C. Kerr, Human Induced Loading on Staircases, PhD. Thesis, University of London, London, UK, 1998.

[22] S. Zivanovic, A. Pavic, and P. Reynolds, "Probability-based prediction of multi-mode vibration response to walking excitation," Engineering Structures, vol. 29, no. 6, pp. 942-954, 2007.

[23] P. Young, "Improved floor vibration prediction methodologies," ARUP Vibration Seminar, vol. 4, 2001.

[24] E. Shahabpoor and A. Pavic, "Measurement of walking ground reactions in real-life environments: a systematic review of techniques and technologies," Sensors, vol. 17, no. 9, paper ID. 2085, 2017.

[25] A. Ancillao, S. Tedesco, J. Barton et al., "Indirect measurement of ground reaction forces and moments by means of wearable inertial sensors: a systematic review," Sensors, vol. 18 , no. 8 , paper ID. 2564, 2018.

[26] International Organization for Standardization, ISO 5982, 2001. 